\title{
Experimental study on the microstructure and nanomechanical properties of the wing membrane of dragonfly
}

\author{
Kewei Xiao · Ke Bai · Wensheng Wang • Fan Song
}

Received: 28 August 2006 / Accepted: 8 November 2006 / Published online: 22 May 2007

(C) Springer-Verlag 2007

\begin{abstract}
Detailed investigations on the microstructure and the mechanical properties of the wing membrane of the dragonfly are carried out. It is found that in the direction of the thickness the membrane was divided into three layers rather than a single entity as traditionally considered, and on the surfaces the membrane displays a random distribution rough microstructure that is composed of numerous nanometer scale columns coated by the cuticle wax secreted. The characteristics of the surface structure are measured and described. The mechanical properties of the membranes taken separately from the wings of live and dead dragonflies are investigated by the nanoindentation technique. The Young's moduli obtained here are approximately two times greater than the previous result, and the reasons that yield the difference are discussed.
\end{abstract}

Keywords Microstructure - Surface structure . Mechanical properties $\cdot$ Surface analysis

The project supported by the National Natural Science Foundation of China (10372102 and 10672164).

K. Xiao · F. Song $(\varangle)$

State Key Laboratory of Nonlinear Mechanics (LNM),

Institute of Mechanics, Chinese Academy of Sciences,

Beijing 100080, China

e-mail: songf@lnm.imech.ac.cn

K. Bai

Department of Chemical Biology,

School of Pharmaceutical Sciences,

Peking University Health Science Center,

Beijing 100083, China

W. Wang

Department of Mechanical Engineering,

Tongji University, Shanghai 200092, China

\section{Introduction}

The wing membrane of insects, which material and mechanical properties are closely associated with the flight capacity of the insects themselves [1,2], is a natural biological membrane made mainly from structural proteins. Most membranes of insect wings undergo significant bending and twisting during flight, which may alter the direction and magnitude of aerodynamic force production [1]. The deformations of the wing membranes increase thrust production in some species by creating a force asymmetry between half-strokes, and can enhance lift production by allowing wings to twist and generate upward force throughout the stroke cycle [3]. Therefore, the material and mechanical properties of the wing membrane, together with that of the wing vein, determine how the wing will change shape in response to these forces [4]. In addition, the membrane is not simply a barrier to the passage of air through the wing but, in some areas at least, has a structural role as a stressed skin, stiffening the framework of veins. And there may be local variation in the mechanical properties and, hence, in the structure of the membrane within the wing, with profound implications for its functioning in flight $[3,4]$. However, the basic material and mechanical properties of the membrane of the insect wings, as well as its microstructure, have not been understood very well [1-5]. For example, the accurate measurements on the mechanical properties of the membrane of the dragonfly wings have remained unclear so far, although there have recently been a lot of investigations on the flight mechanism of the dragonfly and its applications to the micro air vehicles [6,7]. The average Young's module of the wing membrane of dragonflies was measured by the traditional tensile test method to be about $1 \mathrm{GPa}$ [8]. By the nanoindentation technique, the Young's module of the membrane was determined as $1.5 \mathrm{GPa}$ [9]. However, in the recent studies, it is indicated that the rigidity of the wing is a little 
soft so that the insect would have difficulty flying rapidly and agilely in the air when the Young's module of the wing membrane is deemed to be $1.5 \mathrm{GPa}[6,7]$.

Traditionally, the measurements of the mechanical properties of the wing membrane of insects are mainly carried out by using the method of tensile test $[4,5,8]$. However, the tensile test is very inconvenient to some small scale samples of insect wings, such as a cell (a compartment of membrane between wing veins) of dragonfly wings, the area of which is generally about $1 \mathrm{~mm}^{2}$. Moreover, the wing membranes of many insects are corrugated or pleated, at least in part. For example, those of Odonata are pleated almost throughout [8]. Therefore, it is necessary to find a new test method that can more conveniently and accurately measure the mechanical properties of the small scale samples of insects. The nanoindentation technique is an excellent tool for the study of the mechanical behavior of thin membranes, in particular, when simple tensile tests are very small and difficult to perform [9-12]. The development of the nanoindentation technique has allowed highly localized hardness and modulus measurements on very small material volumes. In principle, if a very sharp tip is used, the contact area between the sample and the tip, and thus the volume of material that is tested, can be made arbitrarily small [10,13].

In the present study, we focus on the microstructure and material properties of the wing membrane of the dragonfly, in particular, the differences of mechanical properties between the wing membranes of live and dead dragonflies. By means of scanning electron microscope (SEM), we measure the relevant geometrical and morphological characteristics of the microstructure of the membrane. According to microstructural characteristics, the Young's modulus and the hardness of the membrane are accurately measured by using the nanoindentation technique. Finally, we briefly gave some discussions on the microstructure and material characteristics of the membrane.

\section{Experiments}

\subsection{Materials and sample preparation}

Test samples of the wing membranes were taken from the dragonflies (Libellula basilinea McLachlan) that were caught in the suburb of Beijing. Each of the tested samples consisted only of one cell in the wings of dragonflies. In our investigations, 15 different types of cells taking from 20 forewings of ten dragonflies were tested, and each type of these cells included 20 testing samples in which the live and the dead were separately ten samples. The dead samples of the wing membranes were cut out from the wings of the dragonflies that had died before 3 days and were preserved in a container with room temperature and humidity. Because the material properties of the wing membrane change fairly rapidly after being removed from the insect [4], all live specimens of the membranes were directly removed from the wings of living dragonflies, rapidly installed and examined on the relevant machines within $5 \mathrm{~min}$, so that, the tested properties of the membranes were deemed approximately the same as that of the parts of a living dragonfly.

\subsection{Microstructure measurements}

We employed an SEM (Sirion 400NC, FEI, USA) to observe and measure the microstructure of the wing membrane. Since the thickness of the wing membranes was very thin, we dipped the tested samples in liquid nitrogen for a few minutes so that the membranes became very brittle, then, took the samples out of the liquid nitrogen and broke them to obtain fresh and planar fracture cross-sectional surfaces of the membranes in the direction of the thickness. After that, all the treated samples were coated with gold about $8 \mathrm{~nm}$ thick, and then, the samples were observed by using SEM. Finally, all SEM images were analyzed and recorded by employing Image Analysis System (IAS, Image-Pro Plus 4.5, Media Cybernetics).

\subsection{Mechanical testing}

Nanoindentation tests of the wing membranes were carried out by using a MTS Nano Indenter XP (MTS Systems Corp. Oak Ridge, TN, USA) with a Berkovich diamond tip, and the hardness and the Young's modulus of the membranes were obtained by using the continuous stiffness measurement (CSM) option. We chose an aluminium plate as the testing substrate and used an instant adhesive (502 adhesive) to glue the tested sample on it. Note that the adhesive between the tested membrane and the substrate should be paved as thin and uniform as possible for determining accurately the Young's modulus and the hardness of the membrane [14].

According to the theory of nanoindentation test $[10,13,14]$, the reduced modulus of the tested materials is expressed as:

$E_{r}=\frac{\sqrt{\pi}}{2 \beta} \frac{S}{\sqrt{A}}$,

where $S$ is the contact stiffness that is defined by $S=(\mathrm{d} P / \mathrm{d} h)_{\text {unload }}$, and $\beta$ is a coefficient related to the geometry of the tip, $\beta=1.034$ for the Berkovich tip. However, it is usually difficult to obtain the contact stiffness according to the definition. Employing the CSM method, we have:

$S=\left(\frac{1}{\frac{P_{0}}{Z_{0}} \cos \Phi-\left(K_{s}-m \omega^{2}\right)}-\frac{1}{K_{f}}\right)^{-1}$, 
where $P_{0}$ is the amplitude of the harmonic force; $Z_{0}$ is the amplitude of the tip's displacement; $\Phi$ is the phase angle between the displacement and the force. In this equation, $K_{s}$ and $K_{f}$, the corresponding values of the nanoindenter, $m$, the mass of the indentation system, and $\omega$, the angular frequency of the harmonic load, are constants of the machine, and $P_{0}, Z_{0}$ and $\Phi$ are automatically measured and recorded by the nanoindenter. So, the contact stiffness $S$ is determined by Eq. (2).

Usually, the indented area is difficult to be measured by microscope. Thus, the load and displacement during indentation process are recorded and these data are analyzed to obtain the contact area. The contact area, $A$, is related to the contact depth $h_{c}$, which can be written as:

$A=\sum_{n=0}^{8} C_{n} h_{c}^{\frac{1}{2^{n-1}}}$,

where $C_{n}$ are constants that depend on the indenter geometry and are given by calibrating initially a standard sample with known material properties $[15,16]$. In the present study, $C_{0}=21.4, C_{1}=2100$, and take the rests to be zero. In Eq. (3), $h_{c}$ is determined by:

$h_{c}=h_{\max }-\varepsilon \frac{P_{\max }}{S}$.

For the Berkovich tip, $\varepsilon=0.75$ is a constant depending on the indenter geometry. The relationship between indentation load, $P$, and penetration depth, $h$, is as shown in Fig. 1, where the maximum load, $P_{\max }$, and maximum penetration depth, $h_{\max }$, are recorded automatically by the indenter. From Eqs. (1) to (4), we can obtain the reduced modulus $E_{r}$.

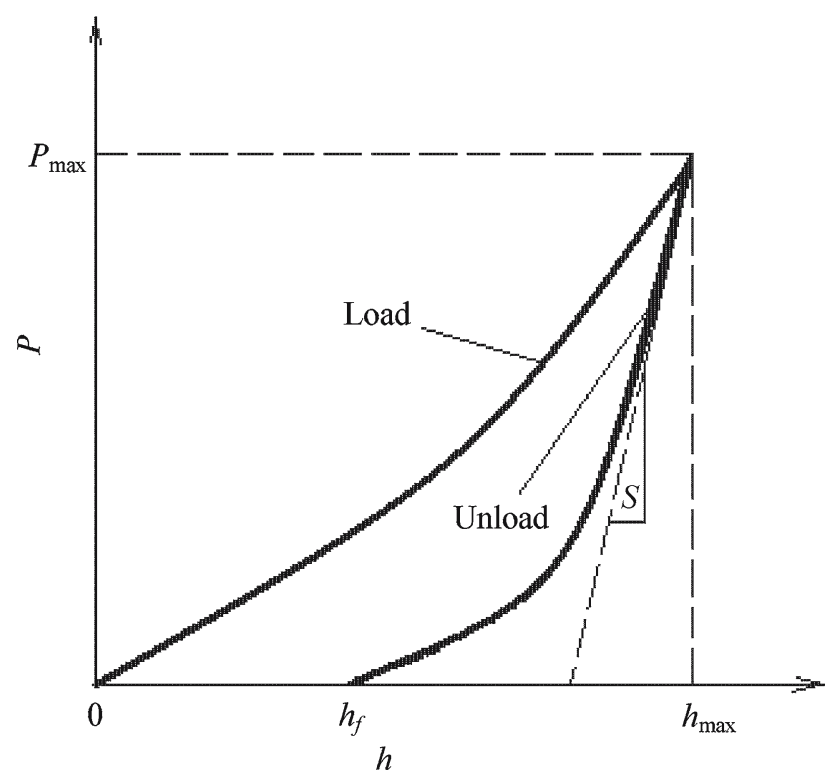

Fig. 1 Plot of indention load, $P$, and penetration depth, $h$, showing the process of the loading and unloading of the nanoindenter
Further, the effects of a non-rigid indenter on the load displacement behavior can be taken into account by defining an effective modulus, $E_{r}$, as follows:

$\frac{1}{E_{r}}=\frac{1-v^{2}}{E}+\frac{1-v_{i}^{2}}{E_{i}}$,

where $E$ is Young's modulus and $v$ is Poisson's ratio of the specimen; $E_{i}$ And $v_{i}$ are the corresponding values of the indenter. For the diamond indenter used in our experiments, $E_{i}=1141 \mathrm{GPa}$ and $v_{i}=0.07$. Also, in the present calculation, Poisson's ratio of the specimen is taken as 0.4 for macromolecular materials [14-16]. The hardness of the material is defined by:

$H=\frac{P_{\max }}{A}$.

According to Eqs. (1)-(6), Young's modulus and the hardness of the tested materials can be obtained. Note that in the testing process of the wing membrane by virtue of CSM method the indenter was firstly loaded to a peak load and held at the peak load for $10 \mathrm{~s}$, then unloaded $90 \%$ from the peak load and held the load for $50 \mathrm{~s}$ for thermal drift correction, finally, unloaded completely. The strain rate and the allowable thermal drift rate were taken in the testing process to be $0.05 \mathrm{~s}^{-1}$ and $0.05 \mathrm{~nm} / \mathrm{s}$, respectively.

\section{Results and discussions}

\subsection{Microstructure tests}

The SEM images of the surfaces of the wing membrane of the dragonfly reveal that along the direction of the thickness the membrane is divided into three layers, which are separately referred to as upper surface, medium layer and lower surface. The thickness of them was measured by the IAS and statistically given to be $(513.63 \pm 69.02) \mathrm{nm},(1.93 \pm 0.18) \mu \mathrm{m}$ and $(356.33 \pm 42.50) \mathrm{nm}$ (mean \pm standard deviation), respectively. And the average gross thickness of the wing membrane was simultaneously measured to be about $(2.8 \pm 0.3) \mu \mathrm{m}$, which was in good agreement with the traditional results [8,9], as shown in Fig. 2a. In addition, both the upper and the lower surfaces, as well as the surface of the wing venations, were found to be composed of numerous nanometer scale rod-like hairs or columns coated by the cuticle wax secreted. These columns almost vertically stood up and randomly distributed on the surfaces of the membranes, and the diameter and the numerical density of the columns on the surfaces were measured to be about $(38.52 \pm 5.88) \mathrm{nm}$ and (75 \pm 7 ) $\mu \mathrm{m}^{-2}$, respectively, as shown in Fig. 2b. From the numerical density, we readily estimated the average distance between two columns on the surface to be about $115 \mathrm{~nm}$. 


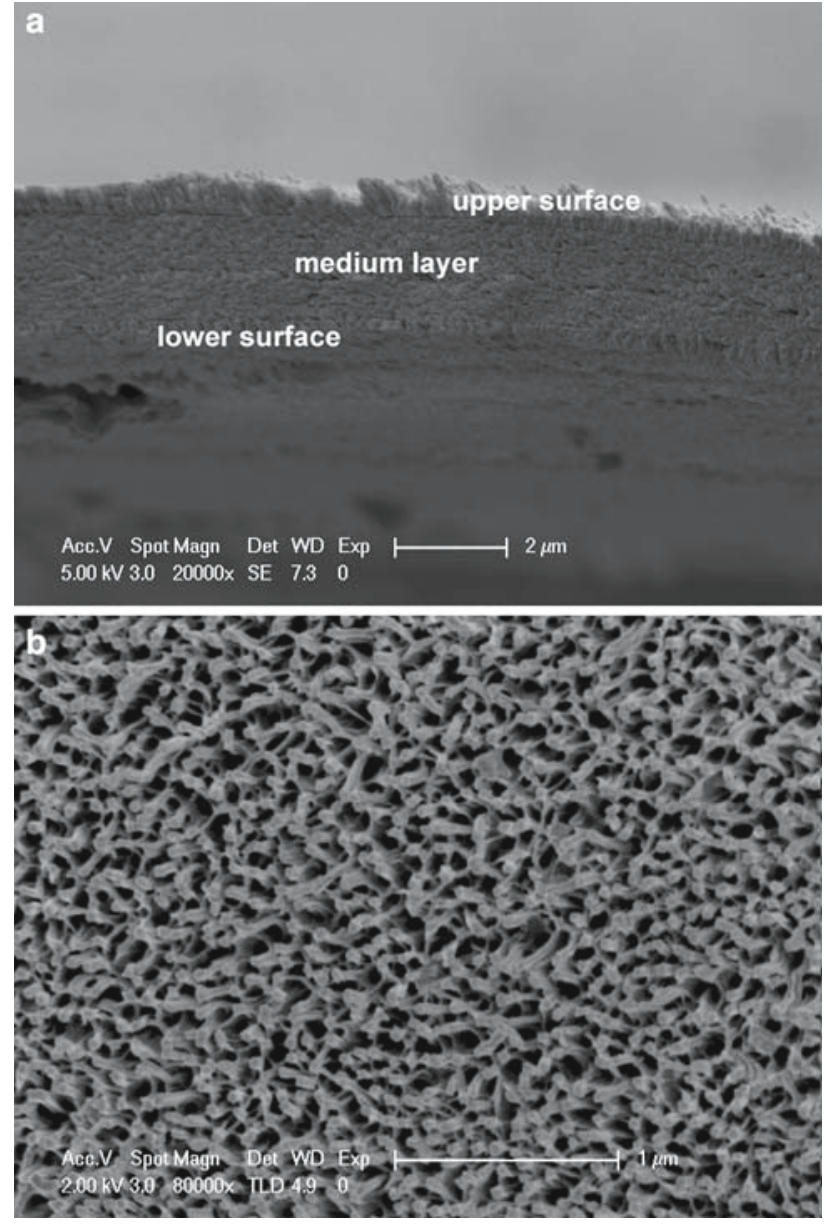

Fig. 2 a The cross-sectional surface of the wing membrane. b The surface view of the cuticle waxy layer

\subsection{Mechanical tests}

According to the microstructure given above, there is a column layer of about $513 \mathrm{~nm}$ thickness on the upper surface of the wing membrane. So, when we employed the indenter to measure the material properties of the membrane, the penetration depth of the tip of the indenter should be greater than the thickness of the column layer. On the other hand, the medium layer and the lower surface of the wing membrane are about 1.93 and $0.36 \mu \mathrm{m}$ thickness, respectively. To ensure that the effects of the substrate are insignificant, the indentation depth should be not greater than $10 \%$ of the tested sample thickness, i.e. $229 \mathrm{~nm}[10,13]$. Therefore, we accurately controlled the indentation depth and only considered the testing data that the indentation depth varied from 550 to $700 \mathrm{~nm}$ as the effective data in the analysis.

According to Eqs. (1) and (6), we readily obtain a relationship between the indentation load and the contact stiffness as:

$$
\frac{P}{S^{2}}=\frac{\pi H}{4 \beta^{2} E_{r}^{2}} .
$$

In Eq. (7), the right hand side of the equation is only composed of material constants. This is an indication that the left hand side of the equation, $P / S^{2}$, is thoroughly determined by the material constants, which should not change with respect to the penetration depth $h$. Therefore, Eq. (7) can be employed to check the reliability of the test data and to control the testing systematic errors [13,14].

Figure 3a shows that $P / S^{2}$ is approximately a constant with respect to the indentation depth $h$ in the effective region from 550 to $700 \mathrm{~nm}$, and Fig. 3b shows that $P$ is approximately proportional to $S^{2}$ with the correlation coefficient for line fitting, $R^{2}$, to approximately reach 0.99 . According to Eq. (7), these indicate that the testing data obtained under these conditions are valid for the tested materials. Figures $3 \mathrm{c}$ and $3 \mathrm{~d}$ separately display the relationships of the average tested Young's modulus and the average tested hardness of the wing membranes taken from live and dead dragonflies with respect to the indentation depth from 550 to $700 \mathrm{~nm}$. The experimental results give that the Young's moduli of the membranes of live and dead dragonflies are $(2.85 \pm 0.23)$ and (2.74 \pm 0.28$) \mathrm{GPa}$, respectively, and the hardness of the wing membranes of the live and the dead are $(0.14 \pm 0.04)$ and $(0.10 \pm 0.03) \mathrm{GPa}$, respectively.

The Young's modulus of the wing membranes of both live and dead dragonflies are approximately two times greater than the previous one, $1.5 \mathrm{GPa}$, obtained by the same test method, namely, the nanoindentation technique [9]. The reason is that the effects of the column layer of the wing surface were not considered in the previous measurements. In addition, it was found that the Young's modulus of the wing membrane of the dragonfly was lower than that of locust $(5 \mathrm{GPa})$, $\alpha$-keratin $(4 \mathrm{GPa}), \beta$-keratin $(8-10 \mathrm{GPa})$, lepidopteran silk (10 GPa) [4], and cicada (3.7 GPa) [12], but was much higher than some amorphous protein polymers, e.g., resilin (1.2 MPa) and abductin (4 MPa) [17]. In particular, the present results indicate that there are only a few differences between the wing membranes taken from live and dead dragonflies. However, if we consider the testing deviations derived from the testing system itself, such as the instability of the indenter, we can approximately deem that there are not differences between the material properties of the wing membranes of the live and the dead dragonflies. Moreover, it is a fact that the wings of dead insects are much more brittle than those of the live. This is an indication that the differences between the mechanical properties of the wing of live and dead insects do not derive from their wing membranes.

\section{Conclusions}

The present work reveals the microstructure and material properties of the wing membrane of the dragonfly. In the direction of the thickness, the membrane is divided into three 

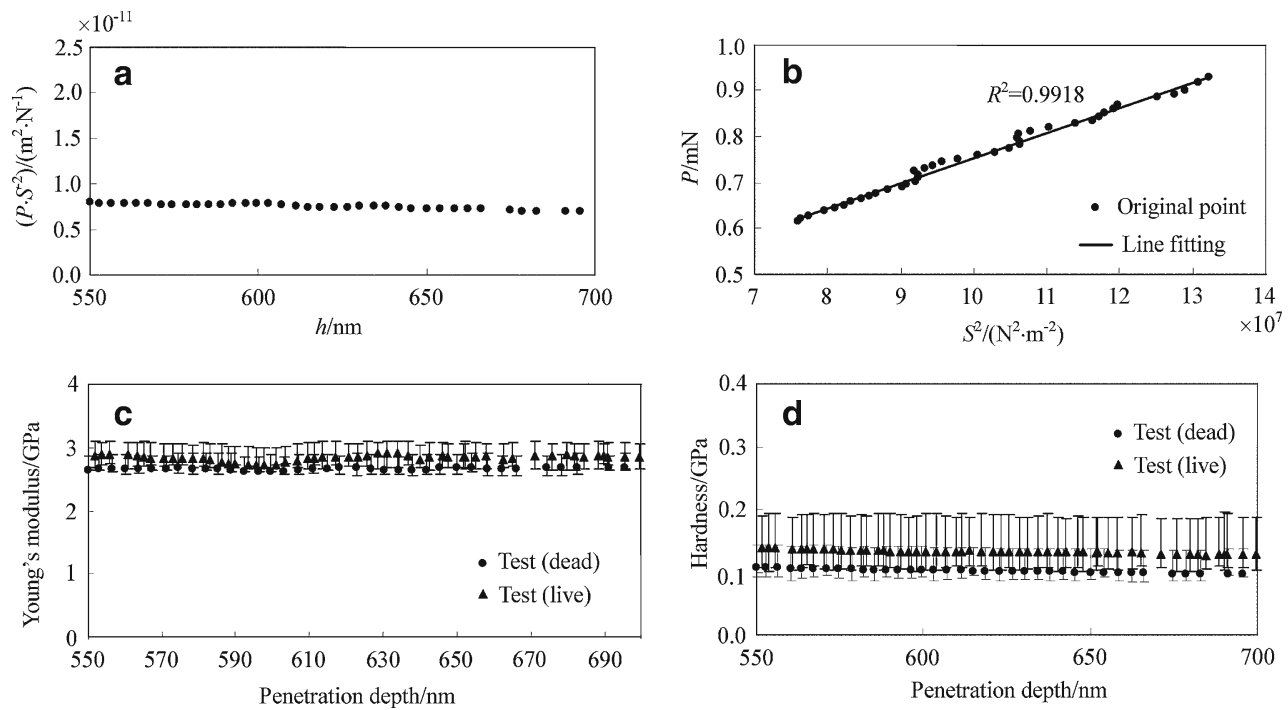

Fig. 3 a The relationship of the quantity, $P / S^{2}$, and the indentation depth, $h$. b The relationship of the indentation load, $P$, and the contact stiffness, $S$. c and $\mathbf{d}$ Tested relationship of the average Young's modulus and the average hardness in the effective region

layers that are separately referred to as upper surface, medium layer and lower surface. Their average thickness is $513.63 \mathrm{~nm}$, $1.93 \mu \mathrm{m}$ and $356.33 \mathrm{~nm}$, respectively. On the upper and lower surfaces, the membrane displays a random distribution rough microstructure that is composed of numerous nanometer scale columns coated by the cuticle wax. The average diameter and numerical density of the columns on the surfaces are about $38.52 \mathrm{~nm}$ and $75 \mu \mathrm{m}^{-2}$, respectively. Accroding to the surface microstructure of the membrane, the average Young's modulus and hardness of the membrane are measured by the nanoindentation technique to be about 2.85 and $0.14 \mathrm{GPa}$, respectively. When we consider the deviations come from the testing system, we indicate that there are few differences of the material properties between the membranes taken from the wings of live and dead dragonflies. The present results can provide a guide to the biomimetic designs of the aerofoil materials of micro air vehicles.

\section{References}

1. Dalton, S.: Borne on the wind: the extraordinary world of insects in flight. Reader's Digest Press, New York (1975)

2. Wootton, R.J.: The mechanical design of insect wings. Sci. Am. 11, 114-120 (1990)

3. Wootton, R.J.: Functional morphology of insect wings. Annu. Rev. Entomol. 37, 113-140 (1992)

4. Smith, C.W., Herbert, R., Wootton, R.J., Evans, K.E.: The hind wing of the desert locust (Schistocerca gregaria Forskål). II. Mechanical properties and functioning of the membrane. J. Exp. Biol. 203, 2933-2943 (2000)

5. Combes, S.A., Daniel, T.L.: Flexural stiffness in insect wings I. Scaling and the influence of wing venation. J. Exp. Biol. 206, 2979-2987 (2003)
6. Ho, S., Nassaf, H., Pornsin-Sirirak, N., Tai, Y.C., Ho, C.M.: Flight dynamics of small vehicles. In: Grant, I. (ed.) Proceedings of the international council of the aeronautical sciences (ICAS)., pp. 551.1-551.10, ICAS, Toronto, Canada (2002)

7. Fearing, R.S., Chiang, K.H., Dickinson, M.H., Pick, D.L., Sitti, M., Yan, J.: Wing transmission of a micromechanical flying insect. In: Alessandro D.L. (ed.) Proceedings of the 2000, IEEE. international conference on robotics and automation, pp. 1509-1516, Wiley, San Francisco, CA, New Jersey (2000)

8. Newman, D.J.S., Wootton, R.J.: An approach to the mechanics of pleating in dragonfly wings. J. Exp. Biol. 125, 361-372 (1986)

9. Kreuz, P., Kesel, B., Arnold, W., Vehoff, H., Nachtigall, W.: Strukter-und materialanalyse biologischer systeme-die Flugelkutikula der insekten (Odonata, Anisoptera). In: Nachtigall W., Wisser A. (eds.) Biona-Report 14, pp. 16-17, Fischer, Stuttgart (2000)

10. Oliver, W.C., Pharr, G.M.: An improved technique for determining hardness and elastic-modulus using load and displacement sensing indentation experiments. J. Mater. Res. 7, 1564-1583 (1992)

11. Morgen, S.E., Misra, R., Jones, P.: Nanomechanical and surface frictional characteristics of a copolymer based on benzoyl-1, 4-phenylene and 1, 3-phenylene. Polymer 47, 2865-2873 (2006)

12. Song, F., Lee, K.L., Soh, A.K., Zhu, F., Bai, Y.L.: Experimental studies of the material properties of the forewing of cicada (Homóptera, Cicàdidae). J. Exp. Biol. 207, 3035-3042 (2004)

13. Joslin, D.L., Oliver, W.C.: A new method for analyzing data from continuous depth-sensing microindentation tests. J. Mater. Res. 5, 123-126 (1990)

14. Saha, R., Nix, W.D.: Effects of the substrate on the determination of thin film mechanical properties by nanoindentation. Acta Mater. 50, 23-38 (2002)

15. Zhang, T.H., Huan, Y.: Substrate effects on the micro/nanomechanical properties of TiN costings. Tribol. Lett. 17, 911-916 (2004)

16. Zhang, T.H., Huan, Y.: Nanoindentation and nanoscrach behaviors of DLC coatings on different steel substrates. Composites Sci. Technol. 65, 1409-1413 (2005)

17. Gosline, J.M.: The elastic properties of rubber-like proteins and highly extensile tissues. In: Vincent J.F.V., Currey J.D. (eds.) The Mechanical Properties of Biological Materials, pp. 331-358, Cambridge University Press, Cambridge (1988) 